\title{
The role of regression and range effects in determination of the power function for electric shock
}

\author{
DAVID V. CROSS, BERNARD TURSKY, and MILTON LODGE \\ State University of New York at Stony Brook, Stony Brook, New York 11794
}

\begin{abstract}
A scale for the apparent intensity of electric shock applied to the forearm was derived from cross-modality matching functions relating noise level, number, and force of handgrip to both line length and shock. For each response mode, the effects of psychophysical regression were estimated from the line judgments and used to make adjustments in the corresponding shock judgments. For shocks ranging from 1.0 to $5.5 \mathrm{~mA}$, combined estimates of subjective magnitude were found to grow as the 2.26 power of the stimulating current.
\end{abstract}

Electric shock has been used extensively as an aversive stimulus in behavioral research and in clinical studies of human pain but not typically with success in predicting quantitative outcomes (Tursky, 1975). The usefulness of this stimulation technique would be increased and the relations it enters into with other variables better understood if we could establish with confidence and without bias the true psychophysical relationships between the physical intensity of electrical stimulation and the attributes of shock-elicited sensations.

Previous efforts to scale the apparent intensity of electric shock have produced contradictory findings. Although results of different experiments are in accord on the basic finding that subjective estimates of shock magnitude grow approximately as a power function of electric current and that the exponent of this function must be greater than unity, there is disagreement concerning the exact value of the exponent. The issue appears to be whether the correct exponent value is closer to 3.5 or to 1.8 .

Using a small range of stimuli, not exceeding two or three times detection threshold, S. S. Stevens and his co-workers in the Laboratory of Psychophysics at Harvard placed this exponent value at about 3.5 (J. S. Stevens, Mack, \& S. S. Stevens, 1960; S. S. Stevens, 1959; S. S. Stevens, Carton, \& Shickman, 1958). Similar exponent values of 3.5 and 3.7 were reported by Hawkes $(1960 \mathrm{a}, \mathrm{b})$ for magnitude estimation of electric shocks ranging from 1.1 to 2 times threshold levels. Using stimulus ranges 2 to 3 times greater than

This research and the preparation of this report were supported by Grant MH 22296-03 of the United States Public Health Service. The authors wish to thank Hugh and Mary Ann Foley for their assistance in carrying out this project. Address requests for reprints to: Professor David V. Cross, Department of Psychology, State University of New York at Stony Brook, Stony Brook, New York 11794. this, seven different experiments reported by Sternbach and Tursky (1964) produced lower exponents with a median value of 1.83 . Exponent values of 1.81 and 1.54 were obtained by Ekman, Frankenhaeuser, Levander, and Mellis (1964, 1966) when observers were asked to judge the unpleasantness of electric shocks that ranged from 2 to 10 times their individual sensation thresholds.

Evidence for the exponent value of 3.5 or greater comes from experiments in which a small range of stimulus intensities were presented and nearthreshold values were included. Both of these experimental constraints produce biases that typically cause spuriously high exponents (Poulton, 1968). It is possible, therefore, that the value of 3.5 overestimates the true exponent value for electric shock. However, the lower values reported by the other investigators might also be biased, but in the opposite direction, because of another source of systematic error in magnitude estimation, namely, regression bias. This bias arises from a tendency for a person's judgments to regress toward a mean level, thus underestimating large magnitudes and overestimating small ones (S. S. Stevens \& Greenbaum, 1966). This bias occurs in both magnitude estimation and production procedures. The typical effect of regression biases in magnitude estimation is to produce a power function with a lower exponent; in magnitude production, it is the stimulus that is adjusted, and the net effect is an increase in the empirical exponent. The nature of the bias is the same in both procedures, but when the data are plotted in the same system of log-log coordinates, the bias causes the best fitting linear functions to diverge from one another and from the true function. This point is illustrated by one of the experiments reported by Sternbach and Tursky (1964) in which magnitude estimation and magnitude production functions were compared. The estimation method 
produced an exponent value of 1.61, and the production method resulted in an exponent of 2.68. It is likely that the true exponent is within the bounds of these two estimates. But their geometric mean of 2.07 would be a good estimate of this true value only if regression biases were equal in estimation and production, an unsupported assumption.

S. S. Stevens (1971) described a procedure that might give an unbiased estimate of the power function relation between any two arbitrary continua, $A$ and $B$. The procedure calls for the matching of a third continuum, $C$, to each of the other two. The ratio of the exponents of the functions matching $C$ to $A$ and $C$ to $B$ determines the exponent of the function relating $B$ to $A$. The regression bias in adjusting $C$ in both intances cancels out in deriving the function relating $B$ to $A$, providing, of course, that the regression occasioned by adjusting $C$ remains constant when the criterion continuum is changed from $\mathrm{A}$ to $\mathrm{B}$.

Cross (1974) elaborated on this procedure and showed that. if the exponent of the power function relating subjective magnitude and stimulus magnitude for Continuum $B$ has a known value, then $B$ can be used as a calibrating continu um wherewith the bias exhibited in matching $C$ against $B$ can be used to make compensating adjustments in the obtained matches of levels of $C$ against $A$ and thereby better estimates of bias-free scale values for $A$. If more than one continuum is used in the role of $C$, each can be separately calibrated against $B$ and the adjusted measures can be pooled for a more precise determination of scale values for $A$.

In the present study, Continuum $A$ is electric shock, and $B$ is line length. The continuum of length is a convenient choice for B because, when factors such as line orientation and context are held constant, judgments of line length are roughly linear with actual length; when one line is twice as long as another, it generally appears to an observer to be two times as long; and hence, judged length is reported to be a power function of actual length with an exponent equal to 1.0 (S. S. Stevens \& Guirao, 1963; M. A. Teghtsoonian, 1965). Because of this proportionality to number, length could serve in place of numbers as a reference continuum in the direct scaling of sensory modalities with no change in the exponents established for these modalities (Krantz, 1972; S. S. Stevens, 1975). If levels of a third continuum, C, are adjusted to match line lengths, the resulting cross-modality matching functions should be equivalent to a magnitude production function. In the present study, three different modalities are used in the role of the matching continuum, $C$ : (1) force of handgrip, (2) loudness of broad-band noise, and (3) number magnitudes. Since typical exponent values for these response modes have been established (J. C. Stevens, 1974; S. S. Stevens, 1972), significant departures from expected exponent values can be interpreted as consequences of regression biases.

The aim of this study is to construct and provide cross-modality validation of a scale for electric shock that is tree of regression biases, range effects, and threshold anomalies, so that it can serve as a valid representation of the sensed effects of electrical stimulation of the skin.

\section{METHOD}

\section{Subjects}

Thirty undergraduate university students were randomly selected from an available pool of about 100 volunteers, both males and females being sampled in equal number. They were all paid for their participation in the study.

\section{Stimuli}

Electrical stimulation was delivered through a concentric disk electrode (Tursky. Watson, \& O'Connell. 1965) applied to the treated dorsal surface of the free arm. the arm not used in squeezing the hand dynamometer. The stimuli were 1-sec shocks from a $60-\mathrm{Hz}$ constant-current source delivered into an electrode-skin circuit impedance that was maintained at $5.000 \pm 500$ ohms (Tursky \& Watson, 1964). RMS values in milliamperes of the current levels used were 1.0. 1.3. 1.8. 2.4. 3.1. 4.1, and 5.5 .

The line stimuli were rear-projected on a $2 \times 2$ ft screen located approximately $5 \mathrm{ft}$ in front of the subject. The following seven line lengths were used: $0.8,2.0,4.0,5.8,8.4,17.7$, and $37.2 \mathrm{~cm}$.

\section{Response Modes}

Judgments of the apparent magnitudes of the electrical shocks and line lengths were expressed separately in three response modes: (1) force of handgrip, (2) noise production, and (3) magnitude estimation. In each mode, the subject produced a response such that (1) the effort exerted in the required handsqueeze, or (2) the loudness of the adjusted noise. or (3) the numerical judgment in each case matched or was proportional to the magnitude of the stimulus being judged.

A standard $1(0)-\mathrm{kg}$ hand dynamometer (Lafayette Instruments) was used to produce the squeeze response. The device consisted of a strong calibrated spring and an attached handle that could be adjusted to each subject's hand size. Built into the dynamometer was a potentiometer that turned as the spring stretched. thus providing an electrical output proportional to the pull exerted on the spring.

Noise levels were adjusted by a sone-potentiometer (Beckman Helipot. NL-5711:600) mounted on the arm of the subject's chair. The sounds produced were intermittent 1 -sec noise bursts (USASI spectrum), which were presented binaurally through calibrated and matching earphones (TDH 39). The source was a noise generator (General Radio, Model 1382). Noise could be heard by the subject only during trials when a noise response was required. The experimenter interposed varying amounts of attenuation between the noise generator and the subject's potentiometer over different trials to insure that the subject's judgments were expressed in terms of noise level rather than potentiometer knob position. The maximum noise level the subject could produce was $100 \mathrm{~dB}$ SPL.

Numerical judgments were expressed vocally. A free magnitude estimation procedure was used (no standard stimulus or modular response). The subject was told that the first number he matched to a stimulus (shock or line) was arbitrary but that successive judgments must reflect the proportional relations that obtained among the perceived shock intensities or observed line lengths. For the handgrip response and noise productions, a standard shock of $2.4 \mathrm{~mA}$ was presented, and the subject was instructed to produce a 
"comfortable" handsquetze or loudness. The only constraint on the subject's choice was that he allow a range of estimates (productions) above and below that assigned to the standard.

\section{Procedure}

During the initial $10 \mathrm{~min}$ of the $60-\mathrm{min}$ session, the subject was given practice squeezing the hand dynamometer, adjusting noise levels, and producing numerical estimates, each time in response to a suggested proportional relation to a modulus value. This continued until the subject expressed confidence in his ability to produce a response in each modality that was proportional to an arbitrary modulus. The subject was then instructed that these response modalities were to be used in making line length estimates by producing. on cue, one of the three responses to each presentation of a line length, observing the requirement that the magnitude of each response be proportional to the perceived length.

Below the projection screen were three panels, one labeled "talk." another "squeeze." and the third, "noise." Two seconds before each stimulus presentation, one of the panels was transilluminated to instruct the subject concerning which of the three responses to make to that stimulus. The response modes were intermingled during the presentation of the six line lengths, which were projected three times each in random order following an initial viewing period in which the subject was shown the full range and sampling of line lengths to be judged.

For the final stage of the session, the instructions to the subject were repeated with the substitution of "shock intensity" for "line length." The seven shock levels were then presented three times each in random order accompanied by a "talk," "squeeze," or "noise" response cue. This procedure insured that each subject produced a squeeze, noise, and number response for each line length and shock stimulus.

\section{Results}

Geometric means of the noise levels, magnitude estimations, and handgrip responses matched to each shock intensity are plotted separately in ratio ruled coordinates in Figure 1. The abscissas are plotted on separate log-cycles for purposes of clarity, and the units for the three response measures were arbitrarily chosen so that the ordinates could be plotted as shown. The relative magnitudes on both axes remain unchanged.

These cross-modality matching functions are well described by power functions. The percent of total variance in $\log$ noise level accounted for by linear regression on log shock intensity is $99.8 \%$. For the other two response measures, the best fitting power functions account for $96.3 \%$ and $97.8 \%$ of total response variance in $\log$ units for magnitude estimation and force of handgrip, respectively.

The lines drawn through the plots in Figure 1 represent the best fitting power functions according to the criterion of least squares in $\log$-log coordinates. The exponents for these power functions are given in Table 1 along with the $95 \%$ confidence limits for the true values.

The cross-modality matching functions relating these response modes to line length are shown in Figure 2. Again, power functions appear to describe these relations fairly well. Linear regression in log-log coordinates accounts for $99.8 \%, 99.7 \%$, and $93.4 \%$

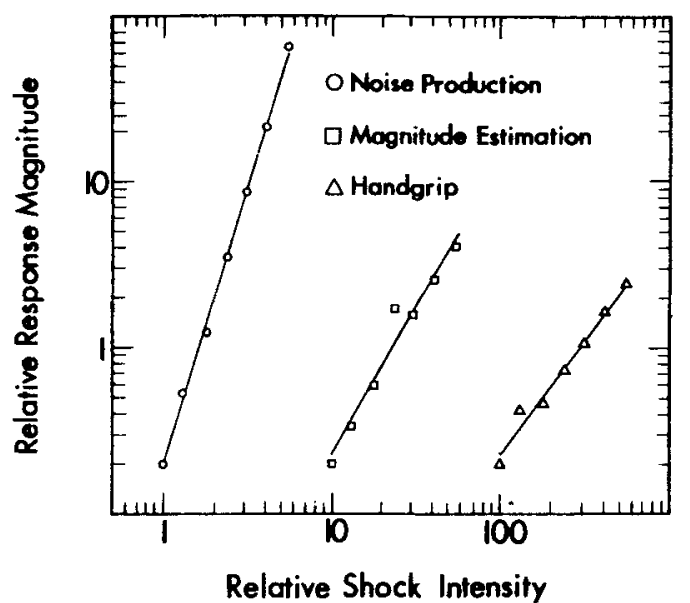

Figure 1. Cross-modality matching functions relating noise level, number, and force of handgrip to electric shock. Each point is the geometric mean of 30 independent responses, one from each subject. The circles stand for noise production, the squares for magnitude estimation, and the triangles for force of handgrip. The abscissas for the three functions are plotted in separate log cycles, and the response units are arbitrary. The slopes of the lines, which give the exponents of the best fitting power functions, were estimated by the method of least squares. The exponent for noise production is 3.35 , the exponent for magnitude estimation is 1.75 , and the exponent for handgrip is 1.39 .

of the variance in the dependent variable, the poorest fit being that for handgrip. Estimates of the exponents for these power functions and $95 \%$ confidence limits for their true values are given in Table 1.

\section{Regression Bias}

The amount of regression bias contained in these empirical exponents can be estimated from the cross-modality matching relation between each response mode and line length. These are equivalent to magnitude production functions for which line length serves in place of number as the criterion continuum. The reciprocals of the exponent values for the power functions fitted to noise production and handgrip vs. line length are 0.68 for loudness and 1.70 for force of handgrip. These exponent values are

Table 1

Point Estimates and 95\% Confidence Limits for Exponents of the Power Functions Relating Noise Level, Number Magnitude, and Force of Handgrip to Shock Intensity and Line Length With Estimates of the Exponent for the Subjective Magnitude of Shock Derived from Each Response Mode as the Ratio of Corresponding Electric Shock to Line Length Exponents

\begin{tabular}{|c|c|c|c|}
\hline \multirow[b]{2}{*}{ Response Modality } & \multicolumn{3}{|c|}{ Stimulus Modality } \\
\hline & $\begin{array}{l}\text { Electric } \\
\text { Shock (a) }\end{array}$ & $\begin{array}{c}\text { Line } \\
\text { Length (b) }\end{array}$ & Ratio $\mathrm{a} / \mathrm{b}$ \\
\hline Noise Production & $3.35 \pm .17$ & $1.46 \pm .08$ & $2.30 \pm .17$ \\
\hline Magnitude Estimation & $1.75 \pm .40$ & $.83 \pm .05$ & $2.11 \pm .50$ \\
\hline Force of Handgrip & $1.39 \pm .24$ & $.59 \pm .18$ & $2.37 \pm .84$ \\
\hline
\end{tabular}




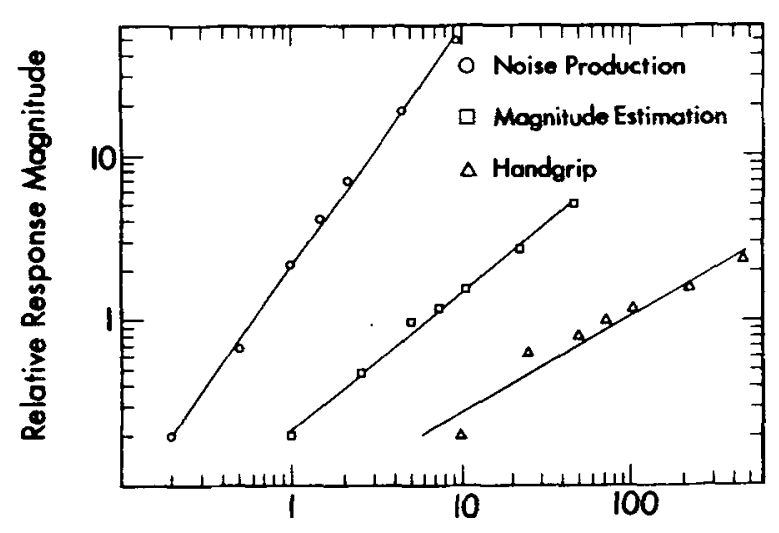

Relative Line Length

Figure 2. Cross-modality matching functions relating noise level, number, and force of handgrip to line length. Each point is the geometric mean of 30 independent responses, one from each subject. The circles stand for noise production, the squares for magnitude estimation, and the triangles for force of handgrip. The abscissas for the three functions are plotted on separate log cycles, and the response units are arbitrary. The slopes of the lines, which give the exponents of the best fitting power functions, were estimated by the method of least squares. The exponent for noise production is 1.46 , the exponent for magnitude estimation is $\mathbf{0 . 8 3}$, and the exponent for handgrip is $\mathbf{0 . 5 9}$.

virtually identical to the current expected values for these modalities when number is used as a reference continuum (J. C. Stevens, 1974; S. S. Stevens, 1972, 1975). This outcome implies that regression biases are negligible in the noise production and handgrip data. Hcwever, the magnitude estimation judgments were nonlinear with actual length; the obtained exponent of 0.83 is significantly different from the expected value of unity $(\mathrm{p}<.001)$. This discrepancy indicates a substantial regression bias in number matching behavior.

\section{Line Length vs. Shock}

Since each response mode enters a power function relation with both electric shock and line length, it follow's that line length itself can be represented as a power function of shock with an exponent equal to the ratio of the electric shock exponent and the line length exponent for each common response mode. These ratios are shown in Table 1 along with $95 \%$ confidence limits for their true values.

Since the exponent values that enter the numerators and denominators of these ratios are themselves random variables-which means they are subject to error-the exponents estimated by the ratios will not be as precisely measured as exponents obtained directly from a cross-modality matching relation. The estimate of the shock exponent derived from the handgrip matches, for example, is consistent with a true value as low as 1.53 or as high as 3.21 ; the $95 \%$ confidence limits include these values.

A more reliable estimate of the shock exponent can be derived from an analysis of the relation between scale values of subjective shock magnitude as derived from the noise productions, handgrip responses, and nunerical judgments for each shock intensity and the corresponding physical measures of shock intensity. The estimated scale values take the form of a weighted geometric mean of the three response measures. For the group data in this experiment, the scaled subjective magnitude of a shock with strength $S_{i}$ is given by

$$
\psi\left(\mathrm{S}_{\mathrm{i}}\right)=\left[\mathrm{P}_{\mathrm{i}}^{0.68} \mathrm{~F}_{\mathrm{i}}^{1.70} \mathrm{~N}_{\mathrm{i}}^{1.20}\right]^{1 / 3},
$$

where $\mathrm{P}_{\mathbf{i}}, \mathrm{F}_{\mathbf{i}}$, and $\mathrm{N}_{\mathbf{i}}$ denote the average noise level (in units of sound pressure), the average force of handgrip (in newtons), and the average number matched to shock, $S_{i}$. The exponents are the reciprocals of the empirical exponent values obtained when each response modality was matched to line length. Using the empirical exponents in this equation rather than the theoretical values presumed to hold for these modalities has the effect of counteracting regression bias in the data. The average response measures are thus transformed into psychological units of loudness, effort. and number magnitude adjusted for regression bias. The composite scale value is obtained as the geometric mean of the three component scale values. The rationale for this procedure has been explained in an earlier report (Cross. 1974).

The scale values calculated with the formula given above are plotted in Figure 3 as a function of $S_{i}$. shock intensity. The curve drawn through the points is

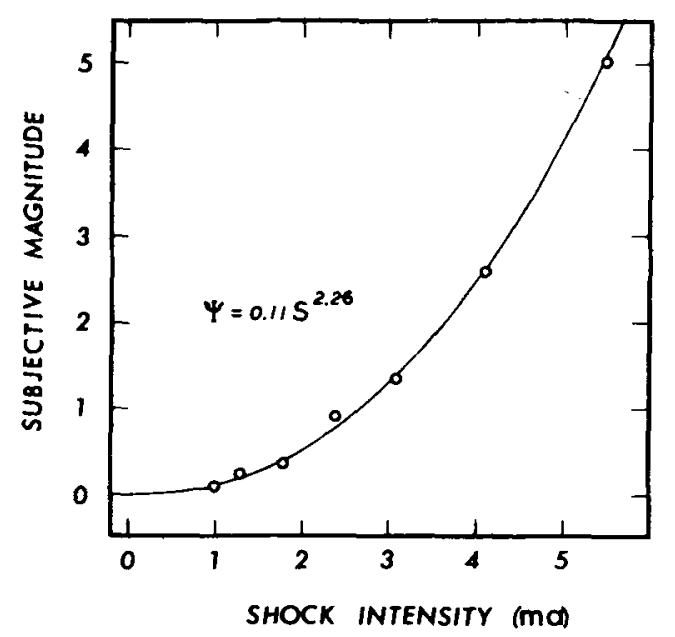

Figure 3. A psychophysical function showing how the perceived magnitude of electric shock applied to the forearm increases with increase in the stimulating current. Each point represents a weighted geometric mean of $\mathbf{3 0}$ noise productions, 30 magnitude estimations, and 30 handgrip responses. The weights were derived from the exponents of the cross-modality matching functions for line length shown in Figure 2. The curve is a graph of the equation shown in the figure; its parameters were estimated by the method of least squares applied to logarithmically transformed data. 
the best fitting power function, the equation for which is indicated in the figure. The function has an exponent value of 2.26 , which happens to be equal to the geometric mean of the three estimates given in table 1 . This is not surprising since both estimation procedures derive from the same set of assumptions regarding the existence and form of regression bias. The standard error of this estimate is 0.088 , and the $95 \%$ confidence limits for the true exponent value are 2.03 and 2.49 .

\section{Individual Exponents}

Individual scales of subjective shock were also constructed for each of the 30 subjects, using individual noise, number, and handsqueeze matches to shock intensity and using the individual exponents determined for each subject's cross-modal matching functions against line length. Functions similar to that depicted in Figure 3 were found for each subject. The distribution of individual exponent values is shown in Figure 4 . While there appears to be considerable variability between subjects-the absolute range goes from 1.65 to 4.08 -some of the most extreme values arise from individuals for whom power functions yielded relatively poor descriptions of the relation between subjective magnitude and shock intensity. In seven cases, indicated by shading of the histogram in Figure 4, from $12 \%$ to $26 \%$ of the variance in $\log \psi$ remained unaccounted for by linear regression on log shock. Most of these individuals had either the largest or the smallest shock exponents. For the remaining 23 cases, $92 \%$ or more of the variance in scale values was explained by the individual power functions in $\log$-log regression. Over half of the 30

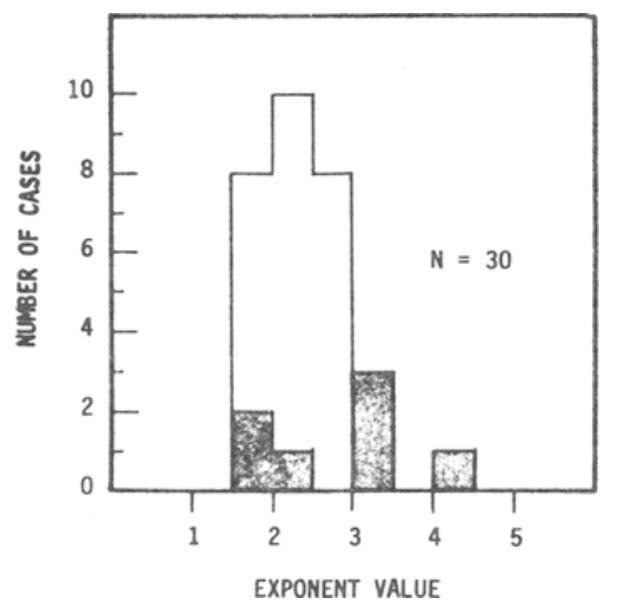

Figure 4. Distribution of exponent values for individual subjects. The exponents belong to separate power functions that were fitted to the composite scale values derived from the cross-modality matching functions for mdividual subjects. The shaded portion of the histogram represents those cases for which the power function gave a relatively poor fit to the data. correlation coefficients were greater than 0.98 . While considerable variability still remains in individual exponents when the seven questionable cases are excluded, the standard deviation expressed as a percentage of the mean (coefficient of variation) is of the same order of magnitude as that established for other modalities after correction for regression effects (S. S. Stevens, 1969).

\section{DISCUSSION}

This study reconfirms the validity of the power law as a description of the relationship between an individual's perception of the strength of an electrical stimulus and its physical intensity. But the characteristic exponent of this relationship appears to be neither as large as the 3.5 that Stevens claimed nor as low as the 1.8 proposed by other investigators. When an adequate stimulus range is used in cross-modality matching procedures that take psychophysical regression into account, an exponent value in the neighborhood of 2.2-2.3 represents the transfer characteristic of cutaneous sensory receptors when they are stimulated by electric current. When shock is expressed in units of electrical power instead of current, this exponent value can be halved. Since the method of stimulation employed in the present study assures a constant electrode skin circuit impedance, the delivered power in watts increases as the square of the delivered current. Thus, under these controlled conditions, it would seem that judgments of the intensity of stimulation are closely associated with delivered power, as suggested by Hill, Flanary, Kornetsky, and Wikler (1952).

Unlike many other stimulus modalities, electricity has no specialized receptor of its own on which to operate, and, for this reason, it has been called the "great inadequate stimulus" (Geldard, 1972). Despite this inadequacy, it is capable of exciting nearly all the senses-possibly by stimulating the nerves directly by alteration of their membrane potentials-hence $S$. S. Stevens (1966) refers to electricity as the "universal stimulus." In the most recent survey of the field (S. S. Stevens, 1975), we find that when the skin is stimulated by a $60-\mathrm{Hz}$ vibration, by static pressure, by irradiation over a limited area, or by an increase in temperature, the sensed effects are power functions with exponents of $0.95,1.1,1.3$, and 1.6, respectively. When rubbing emery cloth, tactual roughness grows as the 1.5 power of grit size. Pain produced by thermal irradiation of the skin grows as a power function with unit exponent. The finding of the present study reveals that, in terms of electrical power, the exponent value of 1.13 for the sensed effects of electrical stimulation of the skin is of the same order of magnitude as the exponents for the other transfer functions of the skin. 
Strictly speaking, we have merely shown that when the judged effects of electric shock are brought into correspondence with equivalent judgments of line length, then line length can be represented as a power function of shock intensity with an exponent of 2.26 . The conclusion that this exponent characterizes the psychological transfer function for electric shock depends on two assumptions: (1) perceived length is proportional to true length, so that line length can serve as a measure of subjective magnitude, and (2) regression biases in magnitude estimation, handsqueeze, and noise productions remain constant when the criterion continuum is changed from shock to line length. This second assumption identifies regression as a response bias that varies, perhaps, with different individual tendencies to avoid extreme judgments or with procedural constraints which limit the range of sensory magnitudes that the subject can produce (for example, the use of a potentiometer that allows the subject to vary noise level over only a $30-\mathrm{dB}$ range). Whatever its actual origin, it is presumed that the regression bias is independent of the stimulus variable. We know, in particular, that this assumption can be violated when assimilative order effects are present in the data (Cross. 1973) or when range effects are present ( $R$. Teghtsoonian, 1973). The experimental design employed in the present study does not permit an independent determination of these effects, so the extent to which regression biases might be different for our shock and line stimuli is unknown. A unique contribution to regression in line judgments by an assimilative order effect that biases the estimated exponent by as little as $10 \%$ could produce the results obtained here with a true exponent for electric shock equal to exactly 2.0 , so our results do not rule out the possibility that perceived intensity of shock is strictly proportional to delivered power.

The import of this finding is far-reading. Psychologists, behavior therapists, and pain researchers who have previously used arbitrary standards to select stimulus intensities for experimental and therapeutic purposes can now select levels of stimulation with known relative subjective magnitudes.

\section{REFERENCES}

Cross, D. V. Sequential dependencies and regression in psychophysical judgments. Perception \& Psychophysics, 1973. 14. 547.552

Cross, D. V. Some technical notes on psychophysical scaling. In H. Moskowitz, B. Scharf, and J. C. Stevens (Eds.). Sensation and measurement: Papers in honor of $S . S$. Stevens. Dordrecht. The Netherlands: Reidel, 1974.

Ekman, G., Frankenhaeuser, M., Levander, S., \& Mellis, I. Scales of unpleasantness of electrical stimulation. Scandinavian Journal of Psychology. 1964, 5, 257-261.
Ekman, G., Frankenhaeuser, M., Levander, S., \& Mellis, I. The influence of intensity and duration of electrical stimulation (m) subjective variables. Scandinavian Joumal of Psychology. $1966.7,58-64$

Geldard, F. A. The human sense's (2nd ed.). New York: Wiley, 1972.

Hawkes, G. R. Cutaneous communication: Absolute identification of electrical intensity level. Jourmat of Psychology, 1960, 49. 203-212. (a)

Hawkes, G. R. An evaluation of the magnitude estimation technique. Journal of Psychology, 1960. 50. 303-3131 (b)

Hill, H. E., Flanary, H. G., Kornetsky, C. H., \& Wikler, A. Relationship of electrically ind uced pain to the amperage and the wattage of shock stimuli. Joumal of Clinical Investigation. 1952. 31. 261-266.

KRANTZ, D. H. A theory of magnitude estimation and cross. modality matching. Journal of Mathematical Psycholog:. 1972. 9.168-199.

Poulton. E. C. The new psychophysics: Six models for magnitude estimation. Psychological Bulletin, 1968. 69. 1-19.

Sternbach, R. A., \& TURSKY. B. On the psychophysical power function in electric shock. Psychonomic Science. 1964. 1. 217-218.

StEvens. J. C. Psychophysical invariances in proprioception. In F. A. Geldard (Ed.). Conference on vibrotactile communication. Austin. Tex: Psychonomic Society. 1974. Pp. 73-77.

Stevens. J. C., Mack, J. D., \& Stevens. S. S. Growth of sensation on seven continua as measured by force of handyrip. Journal of Experimental Psychology, 1960. 59, 60-67.

Stevens. S. S. Cross-modality validation of subjective scales for loudness. vibration. and electric shock. Joumal of Experimental Psychology, 1959, 57, 201-209.

Stevens. S. S. Transfer functions of the skin and muscle senses. Ciba Foundation Symposium on Touch, heat and pain. London: Churchill, 1966. Pp. 3-17.

Stevens, S. S. On predicting exponents for cross-modality matches. Perception \& Psychophysics, 1969, 6, 252-256.

Stevens. S. S. Issues in psychophysical measurement. Psychologicul Revien. 1971, 78, 426-450.

Stevens, S. S. Perceived level of noise by mark VII and decibels (E). Journal of the Acoustical Society of America. 1972. 51. 272-601.

Stevens. S. S. Psychophysics: Introduction to its perceptual, neurul, and social prospects. New York: Wiley, 1975.

Stevens, S. S., Carton, A. S., \& Shickman, G. M. A scale of apparent intensity of electric shock. Joumal of Experimentul Psychology. 1958. 56. 328-334.

Stevens. S. S.. \& Greenbaum, H. B. Regression effect in psychophysical judgment. Perception \& Psychophysics, 1966. 1. 439-446.

Stevens, S. S., \& Guirao, M. Subjective scaling of length and area and the matching of length to loudness and brightness. Journul of Experimental Psychology, 1963, 66, 177-186.

Teghtsoonian, M. A. The judgment of size. American Journal of Psychology, 1965, 78, 392-402.

TEGHTSOONIAN, R. Range effects in psychophysical scaling and a revision of Stevens' law. American Joumal of Psychology. 1973. 86, 3-27.

TuRsky, B. Laboratory approaches to the study of pain. In D. I. Mostofsky (Ed.), Behavioral control and moditication of physiological activity. New York: Prentice-Hall, 1975 (in press). TURSKY. B.. \& W WTSON, P. D. Controlled physical and subjective intensities of electric shock. Psychophysiology, 1964, 1, 151-162.

Tursky, B., Watson, P. D., \& O'CONNEll, D. N. A concentric shock electrode for pain stimulation. Psychophysiology. 1965 . 1. $296-298$.

(Received for publication December 30, 1974: revision received March 13, 1975.) 